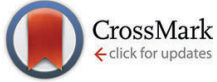

Cite this: Phys. Chem. Chem. Phys., 2016, 18, 18657

Received 9th May 2016 Accepted 15th June 2016 DOI: $10.1039 / c 6 c p 03124 b$

www.rsc.org/pccp

\section{Unusual distance dependences of electron transfer rates}

\begin{abstract}
Martin Kuss-Petermann and Oliver S. Wenger*
Usually the rates for electron transfer $\left(k_{\mathrm{ET}}\right)$ decrease with increasing donor-acceptor distance, but Marcus theory predicts a regime in which $k_{\mathrm{ET}}$ is expected to increase when the transfer distance gets longer. Until recently, experimental evidence for such counter-intuitive behavior had been very limited, and consequently this effect is much less well-known than the Gaussian free energy dependence of electron transfer rates leading to the so-called inverted driving-force effect. This article presents the theoretical concepts that lead to the prediction of electron transfer rate maxima at large donoracceptor distances, and it discusses conditions that are expected to favor experimental observations of such behavior. It continues with a consideration of specific recent examples in which electron transfer rates were observed to increase with increasing donor-acceptor distance, and it closes with a discussion of the importance of this effect in the context of light-to-chemical energy conversion.
\end{abstract}

\section{Introduction}

Due to their low mass, electrons can tunnel over long distances (>15 $\AA$ ). ${ }^{1}$ An exponential decrease of electron transfer rates $\left(k_{\mathrm{ET}}\right)$ with increasing donor-acceptor distance is usually observed for tunneling, governed by the intervening medium as well as the nature of the donor and the acceptor. ${ }^{1 b, 2}$ In cases where the intervening medium contains redox-active units, a so-called hopping mechanism can become operative. ${ }^{3}$ Hopping is essentially multi-step tunneling and consequently leads to shallower distance dependences, typically with $k_{\mathrm{ET}}$ inversely proportional to the donor-acceptor distance. ${ }^{4}$ Regardless of which one of the two mechanisms is active, $k_{\mathrm{ET}}$ usually decreases with increasing reactant separation. However, Marcus theory predicts a regime in which electron transfer rates should first increase to reach a maximum at a given (optimal) donoracceptor distance, and then decrease when increasing the reactant separation even further. ${ }^{5}$ In principle, this was noted more than 30 years ago, ${ }^{6}$ but it remained a largely unknown

Department of Chemistry, University of Basel, St. Johanns-Ring 19, 4056 Basel, Switzerland. E-mail: oliver.wenger@unibas.ch

Martin Kuss-Petermann received his diploma in chemistry from the University of Göttingen (Germany) in 2011 and his PhD degree from the University of Basel (Switzerland) in 2015 under the supervision of Oliver Wenger. His research focusses on phototriggered charge separation and recombination studies, as well as on light-induced charge accumulation. effect, presumably because experimental evidence for it was elusive. Recent studies have provided direct evidence for electron transfer rate maxima at large donor-acceptor distances, ${ }^{7}$ and thus it seemed worthwhile to raise awareness of the underlying theoretical concepts that lead to the prediction of such counterintuitive behavior. We will identify a set of conditions that should favor the observation of increasing $k_{\mathrm{ET}}$ with increasing distance, and we will consider the abovementioned recent studies in this light. Finally, we will discuss the significance of this effect for the conversion of solar light to chemically stored energy.

\section{Basic aspects of electron transfer theory}

According to semi-classical theory, electron transfer rates depend on the reaction free energy $\left(\Delta G_{\mathrm{ET}}^{0}\right)$, the reorganization energy associated with electron transfer $(\lambda)$, and the electronic coupling $\left(H_{\mathrm{DA}}\right)$ between the donor and the acceptor (eqn (1)). ${ }^{5}$

$$
k_{\mathrm{ET}}=\sqrt{\frac{\pi}{\hbar^{2} \cdot \lambda \cdot k_{\mathrm{B}} \cdot T}} \cdot H_{\mathrm{DA}}{ }^{2} \cdot \exp \left(-\frac{\left(\lambda+\Delta G_{\mathrm{ET}}^{0}\right)^{2}}{4 \cdot \lambda \cdot k_{\mathrm{B}} \cdot T}\right)
$$

Oliver S. Wenger received a PhD degree from University of Bern (Switzerland) in 2002 after work with Hans U. Güdel. After two postdocs (one at Caltech with Harry B. Gray from 2002-2004 and one at Université de Strasbourg with Jean-Pierre Sauvage from 20042006) he became assistant professor at University of Geneva. In 2009 he accepted a tenured (W2) professorship at Georg-August-Universität Göttingen, and in 2012 he moved to the University of Basel. 
(a)
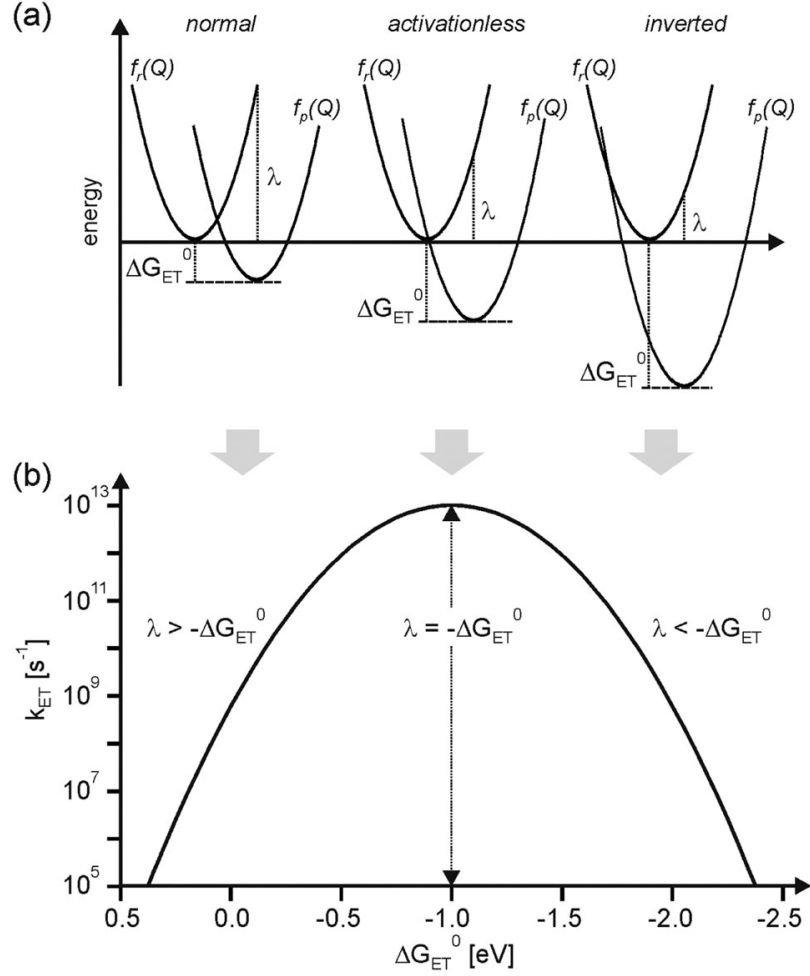

Fig. 1 (a) Reactant $\left(f_{r}(Q)\right)$ and product potential energy wells $\left(f_{p}(Q)\right)$ for electron transfer in three different regimes; (b) dependence of electron transfer rates $\left(k_{E T}\right)$ on reaction free energy $\left(\Delta G_{E T}^{O}\right)$. A reorganization energy (ג) of $1.0 \mathrm{eV}$ was arbitrarily chosen.

The exponential term in eqn (1) is often referred to as the nuclear factor $\left(\kappa_{\mathrm{N}}\right)$, because it captures the effect of nuclear motions occurring in the course of electron transfer. ${ }^{8}$ The remaining factors in eqn (1) can be considered as a product of a frequency factor $\left(\nu_{\mathrm{n}}\right)$ and an electronic factor $\left(\kappa_{\mathrm{e}}\right)$.

The nuclear factor is responsible for the well-known Gaussian free energy dependence of $k_{\mathrm{ET}}$. In the normal regime of the plot in Fig. $1 \mathrm{~b}, k_{\mathrm{ET}}$ increases with increasing driving-force, due to a decreasing activation barrier between the reactant $\left(f_{\mathrm{r}}(Q)\right)$ and product potential energy wells $\left(f_{\mathrm{p}}(Q)\right)$. $k_{\mathrm{ET}}$ reaches a maximum when $-\Delta G_{\mathrm{ET}}^{0}$ is equal to $\lambda$, at which point the reaction proceeds activationless (Fig. 1a, middle). A further increase in drivingforce entails the re-appearance of an activation barrier, leading to a decrease of $k_{\mathrm{ET}}$. This so-called inverted driving-force effect was predicted by theory, ${ }^{5}$ and, after some initial struggle,${ }^{9}$ unambiguous experimental evidence for this phenomenon could be found. Nowadays this effect is well documented and understood. ${ }^{10}$

According to superexchange theory, the electronic coupling term $H_{\mathrm{DA}}$ in eqn (1) can be nonzero even when donor and acceptor are far apart ( $\geq 15 \AA$ A ) because the intervening medium (e.g., covalent bridges or solvent molecules) can mediate longrange electronic coupling. ${ }^{11} H_{\mathrm{DA}}$ usually decreases exponentially with increasing distance $\left(r_{\mathrm{DA}}\right)$, and the steepness of this decrease is captured by the distance decay parameter $\left(\beta_{\mathrm{el}}\right) .{ }^{2 b, 3 a}$

$$
H_{\mathrm{DA}}\left(r_{\mathrm{DA}}\right)=H_{\mathrm{DA}}^{(0)} \cdot \exp \left(-\beta_{\mathrm{el}} \cdot\left(r_{\mathrm{DA}}-r_{\mathrm{DA}}^{(0)}\right)\right)
$$

In eqn (2), $H_{\mathrm{DA}}^{(0)}$ is the electronic coupling between reactants at van-der-Waals contact distance $\left(r_{\mathrm{DA}}^{(0)}\right)$. Exponential distance dependences of $k_{\mathrm{ET}}$ are commonly observed because $k_{\mathrm{ET}} \propto$ $H_{\mathrm{DA}}^{2}$ (eqn (1)), although strictly speaking this is only to be expected for activationless electron transfer $\left(-\Delta G_{\mathrm{ET}}^{0}=\lambda\right.$, see below). Typical distance decay constants $(\beta)$ for $k_{\mathrm{ET}}$ range from $0.4 \AA^{-1}$ for oligo-p-phenylene bridges to $1.1 \AA^{-1}$ for proteins and $1.65 \AA^{-1}$ for water, ${ }^{2 b, 4 b, 12}$ but $\beta$ is governed by the combination of donor, acceptor and intervening medium. ${ }^{2 a, 13}$ Significantly lower $\beta$ values were reported for hopping reactions, ${ }^{14}$ but in such cases the distance decay constant becomes an entirely phenomenological parameter. Note that the distance decay constant $(\beta)$ for $k_{\mathrm{ET}}$ is twice as large as the distance decay constant for $H_{\mathrm{DA}}\left(\beta_{\mathrm{el}}\right.$, eqn (2)) because $k_{\mathrm{ET}} \propto H_{\mathrm{DA}}^{2}$ (eqn (1)).

$H_{\mathrm{DA}}$ is not the only distance-dependent parameter in eqn (1). While the distance dependence of $-\Delta G_{\mathrm{ET}}^{0}$ is often negligible, that of the reorganization energy $(\lambda)$ can be substantial. ${ }^{15}$ The overall reorganization energy is a sum of inner- $\left(\lambda_{i}\right)$ and outersphere $\left(\lambda_{\mathrm{o}}\right)$ contributions (eqn (3)), reflecting the energy required for nuclear reorganization on the donor and the acceptor in the course of electron transfer, as well as reorganization of their chemical environment (e.g., solvent molecules or counter-ions). ${ }^{5}$

$$
\lambda=\lambda_{\mathrm{i}}+\lambda_{\mathrm{o}}
$$

While $\lambda_{\mathrm{i}}$ is commonly treated as a distance-independent parameter, $\lambda_{\mathrm{o}}$ strongly depends on $r_{\mathrm{DA}}$ and on solvent polarity. In the simplest model, the donor and the acceptor are treated as spheres with radii $a_{1}$ and $a_{2}$, separated by the distance $r_{\mathrm{DA}}$ in a solvent with a given optical $\left(D_{\mathrm{op}}\right)$ and static dielectric constant $\left(D_{\mathrm{s}}\right)^{5}$

$$
\lambda_{\mathrm{o}}=\frac{(\Delta e)^{2}}{4 \cdot \pi \cdot \varepsilon_{0}} \cdot\left(\frac{1}{2 \cdot a_{1}}+\frac{1}{2 \cdot a_{2}}-\frac{1}{r_{\mathrm{DA}}}\right) \cdot\left(\frac{1}{D_{\mathrm{op}}}-\frac{1}{D_{\mathrm{s}}}\right)
$$

In eqn (4), $\Delta e$ is the transferred charge, and $D_{\mathrm{op}}$ is related to the refractive index $(\eta)$ by the relationship $D_{\mathrm{op}}=\eta^{2}$. For a donor and an acceptor with radii of $4 \AA$ in $\mathrm{CH}_{3} \mathrm{CN}$, eqn (4) predicts an increase of $\lambda_{\mathrm{o}}$ from $0.94 \mathrm{eV}$ to $1.63 \mathrm{eV}$ when increasing $r_{\mathrm{DA}}$ from contact distance to $30 \AA$ A. More sophisticated models treat the reactants as ellipsoids and permit more precise predictions, ${ }^{16}$ but the key point is that $\lambda_{\mathrm{o}}$ increases with increasing $r_{\mathrm{DA}}$ and thus opposes the distance dependence of $H_{\mathrm{DA}}$.

\section{Consequences of opposing distance dependences of $H_{\mathrm{DA}}$ and $\lambda_{\mathrm{O}}$}

Driving-force dependence parabola such as that in Fig. $1 \mathrm{~b}$ can be calculated as a function of $r_{\mathrm{DA}}$ using eqn (1)-(4). ${ }^{17}$ For spherical donors and acceptors with radii $\left(a_{1}, a_{2}\right)$ of $4 \AA$ in $\mathrm{CH}_{3} \mathrm{CN}\left(\eta=1.3341, D_{\mathrm{s}}=35.7\right)$ at $298 \mathrm{~K}$, we assumed $H_{\mathrm{DA}}^{(0)}=$ $200 \mathrm{~cm}^{-1}, \beta=0.8 \AA^{-1}$, and $\lambda_{\mathrm{i}}=0.1 \mathrm{eV}$. The Marcus parabola obtained for $r_{\mathrm{DA}}=8,11$, and $21 \AA$ are shown in Fig. 2 . The decrease of $H_{\mathrm{DA}}$ with increasing $r_{\mathrm{DA}}$ (eqn (2)) causes a downward shift, while the increase of $\lambda$ (eqn (3) and (4)) displaces the parabola to the right, because the activationless point is reached when $-\Delta G_{\mathrm{ET}}^{0}=\lambda$ (eqn (1)). The two vertical lines in 


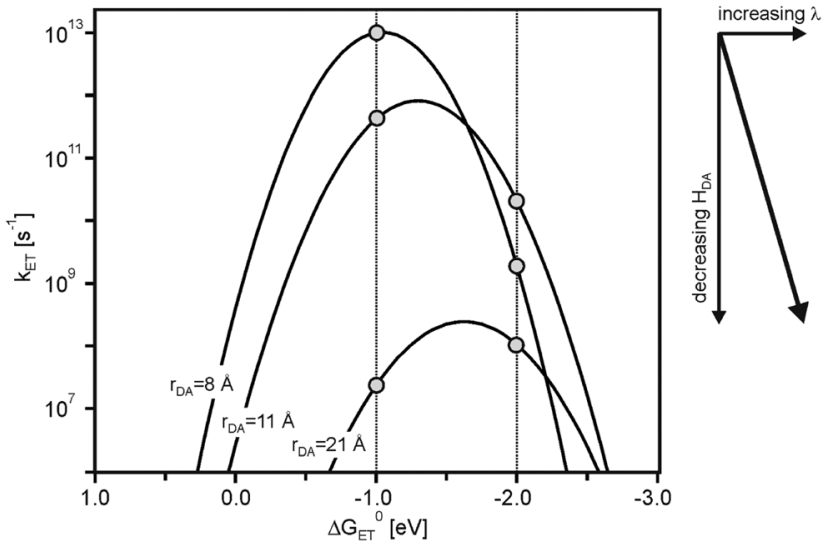

Fig. 2 Driving-force dependence of electron transfer rates $\left(k_{E T}\right)$ at three different donor-acceptor distances $\left(r_{\mathrm{DA}}\right)$. Calculated using eqn (1)-(4) and the following parameters: $H_{\mathrm{DA}}^{(0)}=200 \mathrm{~cm}^{-1}, \beta=0.8 \AA^{-1}, \lambda_{\mathrm{i}}=0.1 \mathrm{eV}, a_{1}=$ $a_{2}=4 \AA, \eta=1.3341\left(D_{\mathrm{op}}=\eta^{2}\right), D_{\mathrm{s}}=35.7$ (values for $\mathrm{CH}_{3} \mathrm{CN}$ ).

Fig. 2 illustrate that at constant driving-force very different distance dependences for $k_{\mathrm{ET}}$ can result, depending on the exact driving-force. At $\Delta G_{\mathrm{ET}}^{0}=-1.0 \mathrm{eV}, k_{\mathrm{ET}}$ decreases with increasing $r_{\mathrm{DA}}$, whereas at $\Delta G_{\mathrm{ET}}^{0}=-2.0 \mathrm{eV}$ there is an increase of $k_{\mathrm{ET}}$ between 8 and $11 \AA$ followed by a decrease at $21 \AA$. In other words, there are regimes in which one expects electron transfer rate maxima at large donor-acceptor distances.

As seen from Fig. 3a, at $\Delta G_{\mathrm{ET}}^{0}=-1.0 \mathrm{eV}$ there is only a minor deviation from strictly exponential dependence of $k_{\mathrm{ET}}$ on $r_{\mathrm{DA}}$, because the distance dependence of the nuclear factor $\left(\kappa_{\mathrm{N}}\right)$ is weak in this case (dotted line), and the contribution from $\nu_{\mathrm{n}} \cdot \kappa_{\mathrm{e}}$ is dominant (dashed line). At $\Delta G_{\mathrm{ET}}^{0}=-2.0 \mathrm{eV}$ (Fig. 3b), however, the distance dependences of $\kappa_{\mathrm{n}}$ and $\nu_{\mathrm{n}} \cdot \kappa_{\mathrm{e}}$ oppose each other, leading to maximal $k_{\mathrm{ET}}$ at $11 \AA$. The reason for this is that the $\left(\lambda+\Delta G_{\mathrm{ET}}^{0}\right)^{2}$ term in eqn (1) decreases with increasing $\lambda$ when $-\Delta G_{\mathrm{ET}}^{0}>\lambda$ (the sum of $\Delta G_{\mathrm{ET}}^{0}$ and $\lambda$ becomes less negative), and this makes the distance dependences of reactions occurring in the inverted driving-force regime fundamentally different from those taking place in the normal regime. From Fig. 2 it is evident that the increase of $k_{\mathrm{ET}}$ with increasing $r_{\mathrm{DA}}$ at relatively short distances relies on a decrease of the ratio $-\Delta G_{\mathrm{ET}}^{0} / \lambda$ from
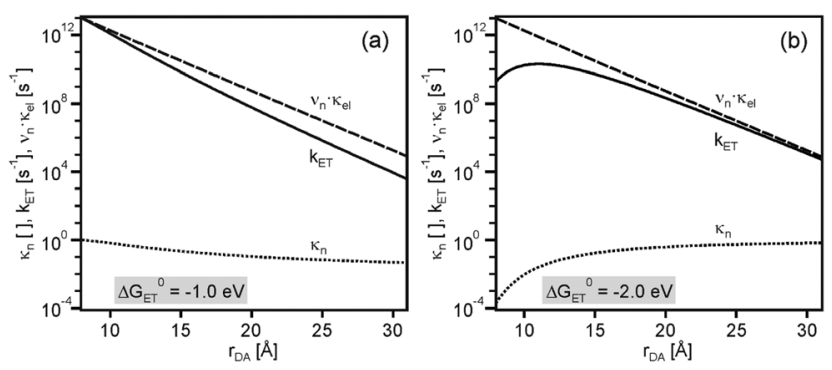

Fig. 3 Distance dependences of electron transfer rates ( $k_{\mathrm{ET}}$, solid lines), the nuclear factor ( $\kappa_{n}$, dotted lines), and the product of frequency factor and electronic factor $\left(\nu_{\mathrm{n}} \cdot \kappa_{\mathrm{e}}\right.$, dashed lines). Calculated using eqn (1)-(4) and the same set of parameters as for Fig. 2, once with $\Delta G_{E T}^{0}=-1.0 \mathrm{eV}$ (a) and once with $\Delta G_{E T}^{0}=-2.0 \mathrm{eV}$ (b). Copyright (a Swiss Chemical Society: CHIMIA 2016, 70, 177; doi: 10.2533/chimia.2016.177. values clearly above 1.0 to values closer to 1.0 . In other words, at $\Delta G_{\mathrm{ET}}^{0}=-2.0 \mathrm{eV}$ the reaction for $r_{\mathrm{DA}}=11 \AA$ is less deeply inverted than for $r_{\mathrm{DA}}=8 \AA$.

While the parameters used above (Fig. 2 and 3) are not unusual in any regard, their choice is somewhat arbitrary. In the following we discuss trends that emerge from variation of these parameters with particular emphasis on identifying conditions that favor the appearance of electron transfer rate maxima at large $r_{\mathrm{DA}}$.

The weaker the contribution of $\nu_{\mathrm{n}} \cdot \kappa_{\mathrm{e}}$ is, the stronger the rate enhancing effect of $\kappa_{\mathrm{n}}$ can become hence low $\beta$ values are favorable. This is illustrated in Fig. 4a which shows the distance dependence of $k_{\mathrm{ET}}$ at $\Delta G_{\mathrm{ET}}^{0}=-2.0 \mathrm{eV}$ for $\beta=0.4,0.8$, and $1.2 \AA^{-1}$ with all other parameters kept identical as in Fig. 3.

An increase in solvent dielectric constant entails larger outer-sphere reorganization energies (eqn (4)), lowering the ratio between $-\Delta G_{\mathrm{ET}}^{0}$ and $\lambda$. As noted above, the increase of $k_{\mathrm{ET}}$ with increasing $r_{\mathrm{DA}}$ at relatively short distances relies on a changeover from deeply inverted to less inverted electron transfer, and if the ratio $-\Delta G_{\mathrm{ET}}^{0} / \lambda$ is close to 1.0 already at contact distance, then expectable effect is less important. Thus, when going from $\mathrm{CH}_{2} \mathrm{Cl}_{2}$ to $\mathrm{CH}_{3} \mathrm{CN}$ and $\mathrm{H}_{2} \mathrm{O}$, the increase of $k_{\mathrm{ET}}$ between contact and optimal distance calculated for $\Delta G_{\mathrm{ET}}^{0}=$ $-2.0 \mathrm{eV}$ with the parameter set from above amounts to factors of 311,11 , and 7, respectively (Fig. 4b). In practice however, a change in solvent will usually lead to changes in both $\Delta G_{\mathrm{ET}}^{0}$ and $\lambda .^{9}$

In a given solvent, an increase in driving-force at constant $\lambda$ amplifies the observable effect because the reaction gets more
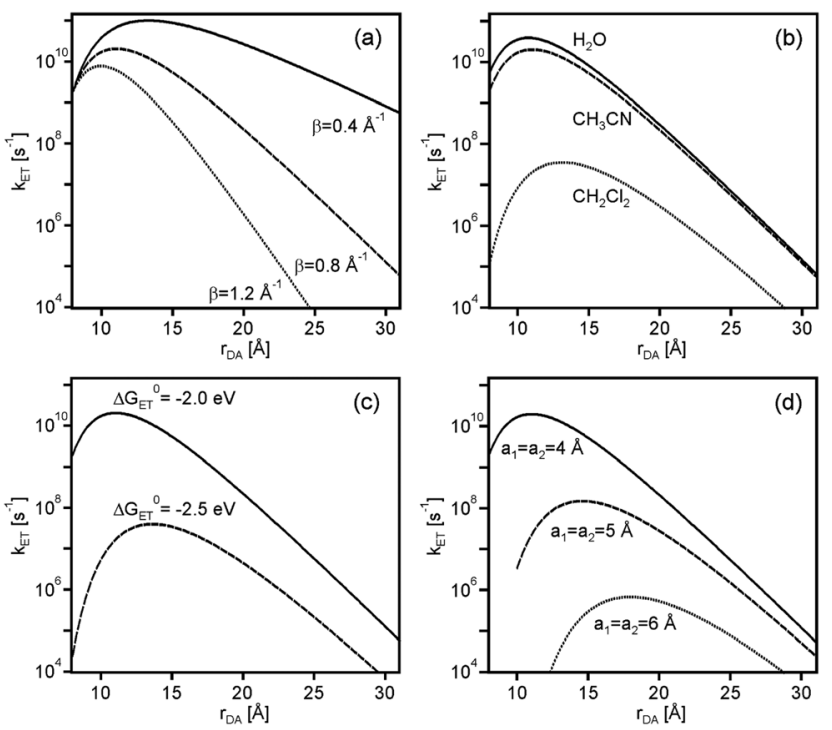

Fig. 4 Distance dependence of $k_{\mathrm{ET}}$ as a function of different parameters: (a) as a function of distance decay constant $(\beta)$; (b) as a function of solvent; (c) as a function of driving-force $\left(\Delta G_{E T}^{O}\right)$; (d) as a function of donor/ acceptor radii $\left(a_{1}, a_{2}\right)$. Unless otherwise noted the calculations were performed for $\mathrm{CH}_{3} \mathrm{CN}\left(\eta=1.3341, D_{\mathrm{s}}=35.7\right) . H_{\mathrm{DA}}^{(0)}=200 \mathrm{~cm}^{-1}$ and $\lambda_{\mathrm{i}}=0.1 \mathrm{eV}$ was used in all cases, the other parameters were as follows: (a) $\Delta G_{\mathrm{ET}}^{0}=-2.0 \mathrm{eV}, a_{1}=a_{2}=4 \AA$; (b) $\Delta G_{\mathrm{ET}}^{0}=-2.0 \mathrm{eV}, a_{1}=a_{2}=4 \AA$, $\beta=0.8 \AA^{-1}$; (c) $a_{1}=a_{2}=4 \AA, \beta=0.8 \AA^{-1}$; (d) $\Delta G_{\mathrm{ET}}^{0}=-2.0 \mathrm{eV}, \beta=0.8 \AA^{-1}$. For the calculations in (b), $\eta=1.3330$ and $D_{\mathrm{s}}=80.1$ were used for $\mathrm{H}_{2} \mathrm{O}$, and $\eta=1.4241$ and $D_{\mathrm{s}}=8.93$ were used for $\mathrm{CH}_{2} \mathrm{Cl}_{2}$. 
deeply inverted. For example, the rate increase between contact and optimal distance in $\mathrm{CH}_{3} \mathrm{CN}$ increases from a factor of 11 to a factor of 1636 when going from $\Delta G_{\mathrm{ET}}^{0}=-2.0 \mathrm{eV}$ to $\Delta G_{\mathrm{ET}}^{0}=$ $-2.5 \mathrm{eV}$ (Fig. 4c), and in $\mathrm{CH}_{2} \mathrm{Cl}_{2}$ the same driving-force change entails an increase in acceleration factor from 311 to $8.08 \times 10^{5}$ when keeping all other parameters constant.

An increase of the donor and acceptor radii $\left(a_{1}, a_{2}\right)$ leads to smaller $\lambda_{\mathrm{o}}$ in a given solvent, making the ratio $-\Delta G_{\mathrm{ET}}^{0} / \lambda$ larger when keeping all other parameters constant. In consequence, for $\Delta G_{\mathrm{ET}}^{0}=-2.0 \mathrm{eV}$ the rate acceleration between contact and optimal distance in $\mathrm{CH}_{3} \mathrm{CN}$ increases by factors of 11,45 , and 146 when increasing $a_{1}$ and $a_{2}$ from 4 to 5 to $6 \AA$ (Fig. $4 \mathrm{~d}$ ).

\section{Influence of nuclear tunneling}

Since the rate maxima at large $r_{\mathrm{DA}}$ rely on reactions which occur in the inverted driving-force regime, nuclear tunneling is expected to influence the magnitude of this effect. Nuclear tunneling relies on the overlap of vibrational wavefunctions between the reactant and product state. In the so-called Jortner model (eqn (5)), this vibrational overlap is captured by the Franck-Condon (FC) factor. ${ }^{18}$

$$
\begin{gathered}
k_{\mathrm{ET}}=\sqrt{\frac{\pi}{\hbar^{2} \cdot \lambda_{\mathrm{o}} \cdot k_{\mathrm{B}} \cdot T}} \cdot H_{\mathrm{DA}}^{2} \cdot(\mathrm{FC}) \\
(\mathrm{FC})=\sum_{\nu^{\prime}} \exp (-S) \cdot \frac{S^{\nu^{\prime}}}{v^{\prime} !} \cdot \exp \left(-\frac{\left(\lambda_{\mathrm{o}}+\nu^{\prime} \cdot \hbar \omega+\Delta G_{\mathrm{ET}}^{0}\right)^{2}}{4 \cdot \lambda_{\mathrm{o}} \cdot k_{\mathrm{B}} \cdot T}\right)
\end{gathered}
$$

In eqn (5), $S$ is the Huang-Rhys parameter describing the displacement of reactant and product potential wells along the reaction coordinate, $\hbar \omega$ is the energy of the vibrational mode responsible for the inner-sphere reorganization occurring with electron transfer, and $\nu^{\prime}$ is the quantum number of the vibrational acceptor level on the product potential energy well. The Huang-Rhys parameter is given by $S=\lambda_{\mathrm{i}} / \hbar \omega$, where $\lambda_{\mathrm{i}}$ is the inner-sphere reorganization energy given as a sum of all the coupled intramolecular vibrations which lead to nuclear rearrangements.

The effect of the Jortner model on the Marcus parabola from Fig. 2 is to make them unsymmetrical, because nuclear tunneling speeds up electron transfer in the inverted region. This is illustrated in Fig. 5a and b (solid lines) where the same parameters as in Fig. 2 were used $\left(\lambda_{i}=0.1 \mathrm{eV}\right)$, but now employing eqn (5) with (a) $\hbar \omega=200 \mathrm{meV}\left(1613 \mathrm{~cm}^{-1}\right)$ and (b) $\hbar \omega=450 \mathrm{meV}\left(3630 \mathrm{~cm}^{-1}\right)$. It is evident from these plots that at constant driving-force, nuclear tunneling weakens the effect of rate maxima at large donor-acceptor separations. This is even more obvious from Fig. $5 \mathrm{c}$ and $\mathrm{d}$ which compares the distance dependences of $k_{\mathrm{ET}}$ in absence (dotted lines) and in presence (solid lines) of nuclear tunneling at different driving-forces. For $\hbar \omega=450 \mathrm{meV}$ (Fig. 5d), the effect of rate maxima at large donor-acceptor separations has disappeared even at $\Delta G_{\mathrm{ET}}^{0}$ as high as $-2.5 \mathrm{eV}$.
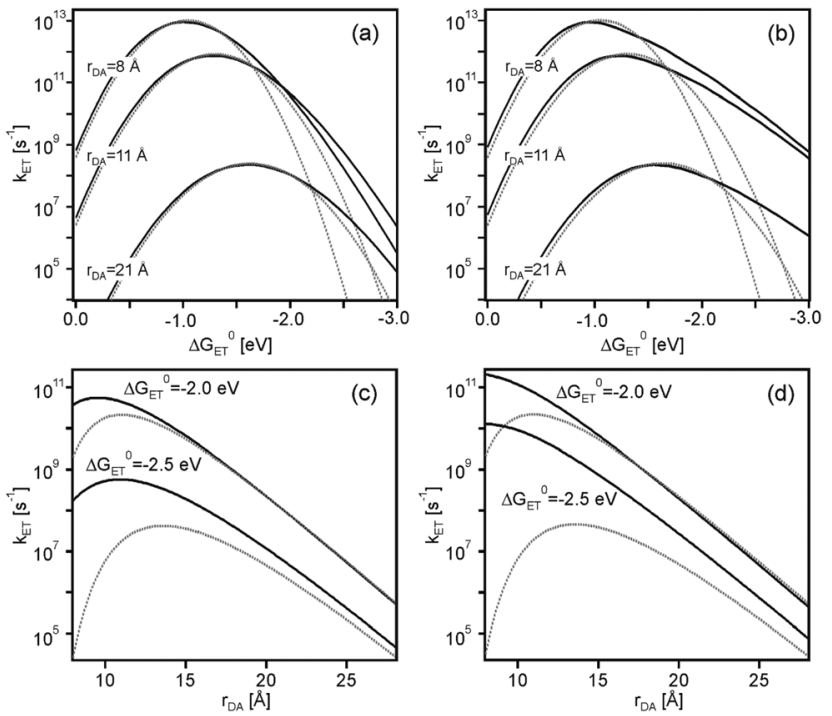

Fig. 5 Effect of nuclear tunneling on driving-force dependences at constant distances ( $a$ and $b$ ) and on distance dependences at constant driving-forces ( $\mathrm{c}$ and $\mathrm{d}$ ) in $\mathrm{CH}_{3} \mathrm{CN}$. The dotted lines in ( $\mathrm{a}$ and $\mathrm{b}$ ) are the same as in Fig. 2, reflecting the situation in absence of nuclear tunneling. The solid lines were calculated using eqn (2)-(5) (i.e., including nuclear tunneling effects) and the following input parameters: $H_{\mathrm{DA}}^{(0)}=200 \mathrm{~cm}^{-1}$, $\beta=0.8 \AA^{-1}, \lambda_{\mathrm{i}}=0.1 \mathrm{eV}, a_{1}=a_{2}=4 \AA$, and (a) $\hbar \omega=200 \mathrm{meV}\left(1613 \mathrm{~cm}^{-1}\right)$ or (b) $\hbar \omega=450 \mathrm{meV}\left(3630 \mathrm{~cm}^{-1}\right)$. Note that $S=\lambda_{\mathrm{i}} / \hbar \omega$. The dotted lines in ( $c$ and d) are the same as in Fig. 4c, reflecting the situation in absence of nuclear tunneling. The solid lines in (c and $d$ ) reflect the situation in presence of nuclear tunneling using the same input parameters as for ( $\mathrm{a}$ and b), once with $\hbar \omega=200 \mathrm{meV}$ (c) and once with $\hbar \omega=450 \mathrm{meV}$ (d).

\section{Experimental observations of increasing electron transfer rates with increasing donor-acceptor distance}

The vast majority of experimental studies reported on electron transfer rates which simply decrease with increasing donoracceptor separation, either due to superexchange tunneling or multi-step hopping. ${ }^{2 a-c, 3 a, 12 a, 13 a, b, 19}$ Some early studies on electron transfer between randomly dispersed donors and acceptors in glassy matrices had invoked the theoretical framework discussed above as a possible explanation for the difficulties associated with observing the inverted driving-force regime in bimolecular electron transfer. ${ }^{20}$ However, to the best of our knowledge, until very recently direct experimental evidence for the effect pointed out in 1984 by Brunschwig, Ehrenson and Sutin had been elusive. ${ }^{6}$

We recently reported on 3 series of donor-photosensitizeracceptor triads in which the rates for thermal electron-hole recombination after initial photoexcitation exhibited maxima at large donor-acceptor distances. ${ }^{7}$ Specifically, the ruthenium(II) photosensitizers of the triads in Fig. 6a were excited selectively at $532 \mathrm{~nm}$, and this lead rapidly to a triarylamine radical cation $\left(\mathrm{TAA}^{+}\right)$and an anthraquinone radical anion $\left(\mathrm{AQ}^{-}\right) .{ }^{21}$ Intramolecular thermal charge recombination was then monitored as a function of distance through variation of the $p$-xylene spacer lengths $(n=1-3)$. The important finding was that the 

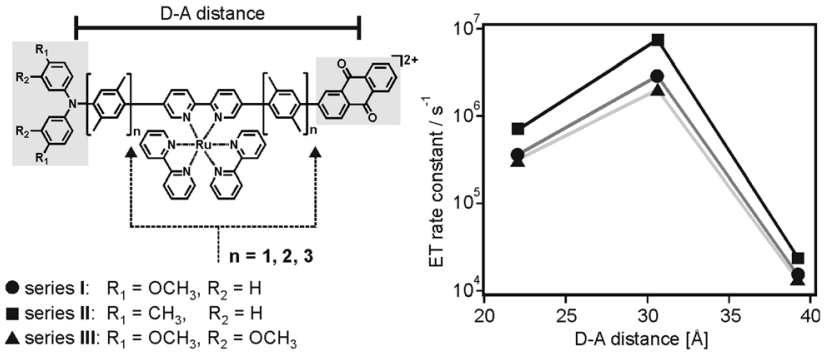

Fig. 6 Chemical structure of donor-photosensitizer-acceptor triads and observation of electron transfer rate maxima as a function of donoracceptor distance. ${ }^{7}$ Reprinted with permission from M. Kuss-Petermann and O. S. Wenger, J. Am. Chem. Soc., 2016, 138, 1349. Copyright 2016 American Chemical Society.

Table 1 Rate constants for electron transfer $\left(k_{\mathrm{ET}}\right)$ from $\mathrm{AQ}^{-}$to $\mathrm{TAA}^{+}$in the three triad series from Fig. 6 in $1: 1(\mathrm{v} / \mathrm{v}) \mathrm{CH}_{3} \mathrm{CN} / \mathrm{H}_{2} \mathrm{O}$ at $20{ }^{\circ} \mathrm{C} .7 \mathrm{n}$ is the number of $p$-xylene bridging units, $r_{\mathrm{DA}}$ is the donor-acceptor distance

\begin{tabular}{|c|c|c|c|c|}
\hline$n$ & $r_{\mathrm{DA}}[\AA]$ & Series I & Series II & Series III \\
\hline 1 & 22.0 & $(3.58 \pm 0.36) \times 10^{5}$ & $(6.90 \pm 0.69) \times 10^{5}$ & $(3.13 \pm 0.31) \times 10^{5}$ \\
\hline 2 & 30.6 & $(2.87 \pm 0.29) \times 10^{6}$ & $(7.41 \pm 0.74) \times 10^{6}$ & $(2.00 \pm 0.20) \times 10^{6}$ \\
\hline 3 & 39.2 & $(1.53 \pm 0.15) \times 10^{4}$ & $(2.43 \pm 0.24) \times 10^{4}$ & $(1.34 \pm 0.13) \times 10^{4}$ \\
\hline
\end{tabular}

rate constant for charge-recombination increased by a factor of 6-10 between the triads with $n=1$ and those with $n=2$, i.e., an increase in donor-acceptor distance by $8.6 \AA$ was accompanied by an increase of $k_{\mathrm{ET}}$ by almost one order of magnitude (right part of Fig. 6). Upon further distance elongation, $k_{\mathrm{ET}}$ then decreased by 2 orders of magnitude (Table 1). In other words, there were rate maxima at a donor-acceptor distance of $30.6 \AA$ A.

This unusual observation was explained in the framework of the model illustrated by Fig. $2{ }^{6}$ Temperature-dependent studies indicated that charge-recombination in the triads with $n=2$ proceeded in activationless manner, whereas in the systems with $n=1$ or $n=3$ this process required significant thermal activation. Keeping in mind that the reorganization energy commonly increases with increasing donor-acceptor distance (eqn (4)), the temperature-dependent studies were interpreted in terms of a changeover from the inverted regime $(n=1)$ to activationless $(n=2)$ and normal electron transfer $(n=3)$. This changeover is illustrated by the potential well diagrams in Fig. 7. The reaction free energy $\left(\Delta G_{\mathrm{ET}}^{0}\right)$ is essentially distance-independent in these systems, but the increase in reorganization energy $(\lambda)$ then leads to decreasing ratios of $-\Delta G_{\mathrm{ET}}^{0} / \lambda$ with increasing distance, manifesting in the abovementioned changeover between different regimes. In the investigated triads $-\Delta G_{\mathrm{ET}}^{0} / \lambda$ varied from $\sim 1.4(n=1)$ to $\sim 0.5(n=3)$.

Two aspects of these studies deserve further comment. First of all, the increase of $\lambda$ was very large, particularly between the triads with $n=2(\lambda=1.3-1.5 \mathrm{eV})$ and $n=3(\lambda=2.0-2.2 \mathrm{eV})$. In principle, reorganization energies up to $\sim 2.0 \mathrm{eV}$ in $\mathrm{CH}_{3} \mathrm{CN} /$ $\mathrm{H}_{2} \mathrm{O}$ mixtures can be explained adequately by the breakage of hydrogen-bonds between anthraquinone radical anion and solvent molecules, ${ }^{22}$ but it is not obvious why the increase between $n=2$ and $n=3$ is so large. Second, the decrease of the

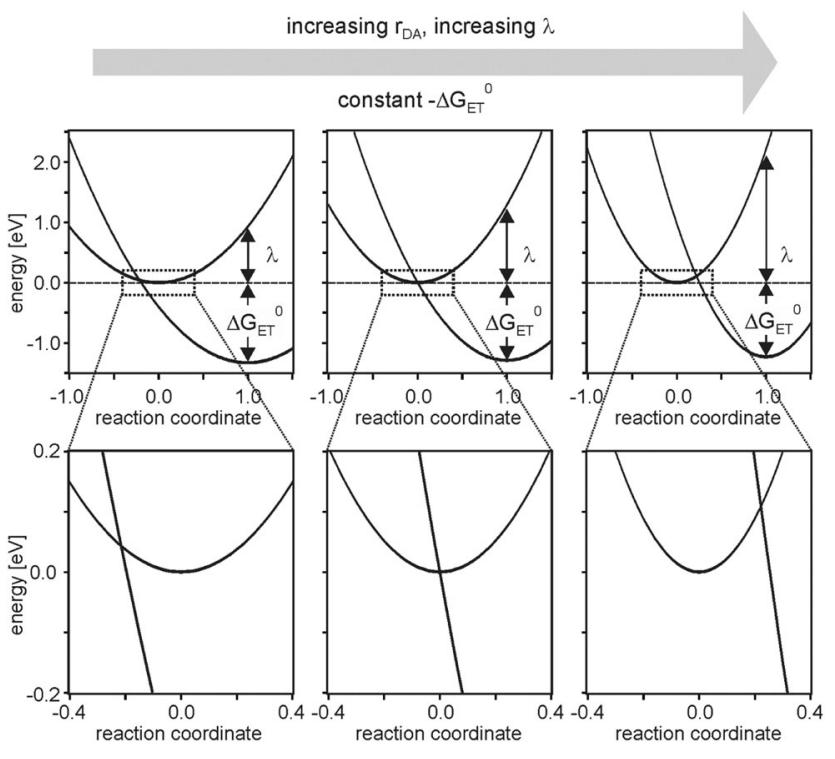

Fig. 7 Harmonic potential energy wells for reactant and product states of charge-recombination between $\mathrm{AQ}^{-}$and $\mathrm{TAA}^{+}$in the triads from Fig. 6 . The lower half shows zooms of the key regions from the upper half. Reprinted with permission from M. Kuss-Petermann and O. S. Wenger, J. Am. Chem. Soc., 2016, 138, 1349. Copyright 2016 American Chemical Society.

electronic coupling matrix element $\left(H_{\mathrm{DA}}\right)$ with increasing distance was extremely shallow, in fact nearly distance-independent for some of the triad systems considered until now. This finding is all the more astonishing in light of prior studies of the distance dependence of electron transfer through oligo- $p$-xylenes which provided $\beta$-values in the range $0.52-0.76 \AA^{-1}$. $^{23}$ It is likely that the $2,2^{\prime}$-bipyridine ligand unit and its coordinated ruthenium(II) complex have a strong influence on the electronic donoracceptor coupling, and there was direct evidence for significant $\pi$-conjugation between this $2,2^{\prime}$-bipyridine ligand and its adjacent $p$-xylene groups. ${ }^{7}$ Nevertheless, the extent to which $H_{\mathrm{DA}}$ is insensitive to distance remains astonishing.

The harmonic potential well model (Fig. 2) used to account for the observation of rate maxima at large donor-acceptor distances is almost certainly too simplistic to grasp the full complexity of the problem, and once more experimental data will be available, then it will be worthwhile considering more sophisticated theoretical treatments. The finding of unusually large reorganization energies and very shallow distance dependences of $H_{\mathrm{DA}}$ might have a common origin, for example it is possible that $\lambda$ and $H_{\mathrm{DA}}$ cannot be considered mutually fully independent parameters in some of these systems.

\section{Consequences for light-to-chemical energy conversion}

For solar energy conversion one is interested in fast (efficient) photoinduced charge-separation combined with slow (inefficient) thermal charge-recombination. ${ }^{28}$ Photoinduced charge-separation reactions commonly occur in the normal regime in which $-\Delta G_{\mathrm{ET}}^{0}<\lambda$. Under these conditions, $k_{\mathrm{ET}}$ simply decreases with 


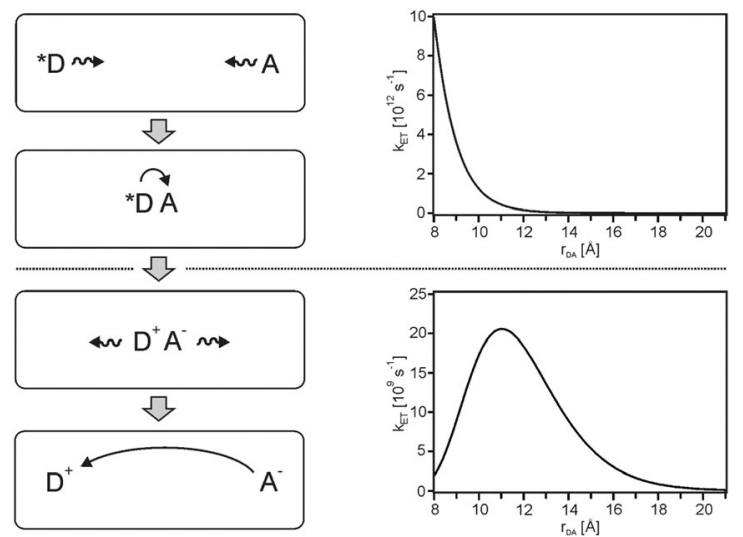

Fig. 8 Left: Diffusive motion leading to the formation of an encounter complex between an excited donor $\left({ }^{*} \mathrm{D}\right)$ and an acceptor (A), followed by photoinduced charge-separation. Diffusion then separates the photoproducts spatially, but since charge-recombination is usually highly exergonic it can exhibit a rate maximum at large distances. Right: Distance dependences for weakly exergonic $\left(\Delta G_{\mathrm{ET}}^{0}=-1.0 \mathrm{eV}\right.$, top) and strongly exergonic reactions ( $\Delta G_{\mathrm{ET}}^{0}=-2.0 \mathrm{eV}$, bottom), calculated using eqn (1) $-(4)$ and the same parameters as in Fig. 2 .

increasing $r_{\mathrm{DA}}$ hence photoinduced charge-separation is fastest between reactants that are in van-der-Waals contact (upper half of Fig. 8). In terms of converting light into chemical energy it is then desirable for the products (oxidized donor, $\mathrm{D}^{+}$, and reduced acceptor, $\mathrm{A}^{-}$) to diffuse away from one another without undergoing direct charge-recombination. However, chargerecombination frequently occurs in the inverted regime in which $-\Delta G_{\mathrm{ET}}^{0}>\lambda$, and consequently the rate for this undesired reaction can actually increase with increasing separation between $\mathrm{D}^{+}$and $\mathrm{A}^{-}$to reach an optimum at a critical distance (lower half of Fig. 8). In other words, as $\mathrm{D}^{+}$and $\mathrm{A}^{-}$diffuse away from each other, the probability for them to undergo undesired charge-recombination actually increases up to a critical distance, and only beyond this point there is a decrease. This effect can severely limit the overall efficiency of light-to-chemical energy conversion.

\section{Nuclear factor contributions to the distance dependence of $k_{\mathrm{ET}}$ in other systems}

Many studies on proteins focused on activationless electron transfer because under this condition it is possible to isolate the contribution of the electronic factor to the distance dependence of $k_{\mathrm{ET}}{ }^{12 b}$ In cases in which the electron transfer is not activationless, electronic and nuclear factors both contribute to the distance dependence of $k_{\mathrm{ET}}$, and there are a few examples in the literature where the contribution of the nuclear factor became particularly evident. Electron transfer across proline bridges in the three series of dyads from Fig. 9 was triggered by pulse radiolysis through reduction of the Os(III) species (Os-Co, Os-Ru series) or the $\mathrm{Ru}(\mathrm{bpy})_{3}{ }^{2+}$ unit (Ru-Co series). ${ }^{24}$ The distance dependence of $k_{\mathrm{ET}}$ deviates significantly from strictly mono-exponential behavior in
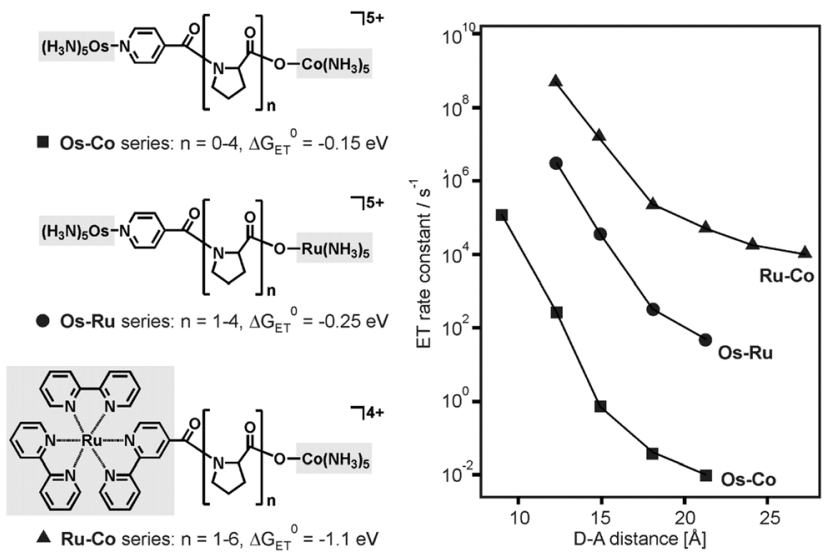

Fig. 9 Donor-acceptor dyads with proline bridges and distance dependences of $k_{\mathrm{ET}}$ after reduction of the donor moieties (left-hand sides) with pulse radiolysis. The change from $\mathrm{Os}-\mathrm{CO}^{25}$ to $\mathrm{Os}-\mathrm{Ru}^{26}$ and $\mathrm{Ru}-\mathrm{Co}$ systems ${ }^{27}$ allowed a change in driving-force $\left(\Delta G_{E T}^{0}\right)$ from -0.15 to $-1.1 \mathrm{eV}$.

all three dyad series, and this was interpreted in terms of strongly distance-dependence nuclear $\left(\kappa_{\mathrm{n}}\right)$ and electronic factors $\left(\kappa_{\mathrm{e}}\right) \cdot{ }^{24}$ Using an approach that expresses the activation parameters for intramolecular electron transfer in terms of transition state theory, activation enthalpies $\left(\Delta H^{\ddagger}\right)$ and activation entropies $\left(\Delta S^{\ddagger}\right)$ were determined. It was then argued that under certain assumptions, ${ }^{15 a}$ the slope of $\Delta S^{\ddagger} / R$ versus distance $(R$ is the universal gas constant) yields the distance decay constant $(\beta)$ of the electronic factor, whereas the slope of $-\Delta H^{\ddagger} / R \cdot T$ versus distance provides the distance decay constant $(\gamma)$ for the nuclear factor. ${ }^{24}$ For the Os-Ru dyads from Fig. 9 the assumptions of this approach were justified, ${ }^{24}$ and the distance dependence of $\kappa_{\mathrm{n}}$ turned out to be stronger than that of $\kappa_{\mathrm{e}}$ $\left(\gamma=0.91 \AA^{-1}\right.$ vs. $\left.\beta=0.68 \AA^{-1}\right) .{ }^{15 a}$ This is a remarkable finding and an instructive example of how important nuclear factor contributions to the distance dependence of $k_{\mathrm{ET}}$ can be. The parallel to our triads is that we also observe strongly distance dependent nuclear factors (Fig. 7).

In the Os-Ru dyads from Fig. 9 the reorganization energy increased from $1.22 \mathrm{eV}(n=1)$ to $1.52 \mathrm{eV}(n=2)$ to finally $1.78 \mathrm{eV}$ $(n=3)$, whereas in the case of our own triads from Fig. 6 the change in $\lambda$ was even larger (0.9-2.2 eV, see above) but this occurred over a significantly greater distance range $(17.2 \AA$ compared to $5.9 \AA$ A). The driving-force associated with intramolecular electron transfer in the Os-Ru dyads is relatively small $\left(\Delta G_{\mathrm{ET}}^{0}=-0.25 \mathrm{eV}\right) \cdot{ }^{26}$ Consequently, a changeover in the ratio between $-\Delta G_{\mathrm{ET}}^{0}$ and $\lambda$ from values above 1.0 (indicative of inverted behavior) to values below 1.0 (signaling electron transfer in the normal regime) was not observable in these systems.

Nevertheless, the dyads from Fig. 9 represent early examples of clear-cut cases in which contributions of the nuclear factor to the distance dependence of $k_{\mathrm{ET}}$ became observable. In other early studies, the model illustrated by Fig. 2 was invoked to explain the difference in the distance dependences of weakly exergonic charge-separation reactions and strong exergonic charge-recombination processes. ${ }^{15 c}$ 


\section{Conclusions}

There are two counter-intuitive scenarios for electron transfer: (i) decreasing rates with increasing driving-force (at constant donor-acceptor distance), and (ii) increasing rates with increasing donor-acceptor distances (at constant driving-force). The first scenario, usually called the inverted driving-force effect, ${ }^{5}$ is very well known and experimentally well documented. ${ }^{10}$ The second scenario, while predicted by theory a long time ago, ${ }^{6}$ is comparatively little known, presumably because unambiguous experimental evidence for it was found only very recently. ${ }^{7}$

Increasing rates with increasing donor-acceptor distances can result from increasing (outer-sphere) reorganization energies $\left(\lambda_{\mathrm{o}}\right)$ in the inverted driving-force regime, leading to lower activation barriers at constant driving-force. Our recent experimental studies demonstrated that it is even possible to induce a changeover from the inverted to the normal regime by increasing the donor-acceptor separation. ${ }^{7}$ When dealing with photoinduced electron transfer reactions, this effect becomes important for light-to-chemical energy conversion.

\section{Acknowledgements}

Financial support by the Swiss National Science Foundation through grant number 200021-146231/1 is gratefully acknowledged.

\section{Notes and references}

1 (a) D. De Vault and B. Chance, Biophys. J., 1966, 6, 825-847; (b) J. R. Winkler and H. B. Gray, J. Am. Chem. Soc., 2014, 136, 2930-2939.

2 (a) B. Albinsson, M. P. Eng, K. Pettersson and M. U. Winters, Phys. Chem. Chem. Phys., 2007, 9, 5847-5864; (b) H. B. Gray and J. R. Winkler, Proc. Natl. Acad. Sci. U. S. A., 2005, 102, 3534-3539; (c) M. Natali, S. Campagna and F. Scandola, Chem. Soc. Rev., 2014, 43, 4005-4018; (d) F. D. Lewis, J. Q. Liu, W. Weigel, W. Rettig, I. V. Kurnikov and D. N. Beratan, Proc. Natl. Acad. Sci. U. S. A., 2002, 99, 12536-12541; (e) O. S. Wenger, Inorg. Chim. Acta, 2011, 374, 3-9; $(f)$ D. C. O'Hanlon, B. W. Cohen, D. B. Moravec, R. F. Dallinger and M. D. Hopkins, J. Am. Chem. Soc., 2014, 136, 3127-3136; (g) A. Helms, D. Heiler and G. McLendon, J. Am. Chem. Soc., 1992, 114, 6227-6238; (h) L. T. Calcaterra, G. L. Closs and J. R. Miller, J. Am. Chem. Soc., 1983, 105, 670-671; (i) H. Oevering, M. N. Paddon-Row, M. Heppener, A. M. Oliver, E. Cotsaris, J. W. Verhoeven and N. S. Hush, J. Am. Chem. Soc., 1987, 109, 3258-3269; ( j) S. V. Rosokha, D.-L. Sun and J. K. Kochi, J. Phys. Chem. A, 2002, 106, 2283-2292.

3 (a) M. Cordes and B. Giese, Chem. Soc. Rev., 2009, 38, 892-901; (b) C. Shih, A. K. Museth, M. Abrahamsson, A. M. Blanco-Rodriguez, A. J. Di Bilio, J. Sudhamsu, B. R. Crane, K. L. Ronayne, M. Towrie, A. Vlček, J. H. Richards, J. R. Winkler and H. B. Gray, Science, 2008, 320, 1760-1762; (c) C. Lambert, G. Nöll and J. Schelter, Nat. Mater., 2002, 1, 69-73; (d) M. Cordes, A. Kottgen, C. Jasper, O. Jacques, H. Boudebous and B. Giese, Angew. Chem., Int. Ed., 2008, 47,
3461-3463; (e) J. C. Genereux and J. K. Barton, Chem. Rev., 2010, 110, 1642-1662; $(f)$ V. Lloveras, J. Vidal-Gancedo, T. M. Figueira-Duarte, J. F. Nierengarten, J. J. Novoa, F. Mota, N. Ventosa, C. Rovira and J. Veciana, J. Am. Chem. Soc., 2011, 133, 5818-5833; $(g)$ K. E. Linton, M. A. Fox, L. O. Palsson and M. R. Bryce, Chem. - Eur. J., 2015, 21, 3997-4007.

4 (a) W. B. Davis, W. A. Svec, M. A. Ratner and M. R. Wasielewski, Nature, 1998, 396, 60-63; (b) O. S. Wenger, Chem. Soc. Rev., 2011, 40, 3538-3550.

5 R. A. Marcus and N. Sutin, Biochim. Biophys. Acta, 1985, 811, 265-322.

6 B. S. Brunschwig, S. Ehrenson and N. Sutin, J. Am. Chem. Soc., 1984, 106, 6858-6859.

7 (a) M. Kuss-Petermann and O. S. Wenger, Angew. Chem., Int. Ed., 2016, 55, 815-819; (b) M. Kuss-Petermann and O. S. Wenger, J. Am. Chem. Soc., 2016, 138, 1349-1358.

8 N. Sutin, Acc. Chem. Res., 1982, 15, 275-282.

9 D. Rehm and A. Weller, Isr. J. Chem., 1970, 8, 259-271.

10 (a) G. L. Closs and J. R. Miller, Science, 1988, 240, 440-447; (b) M. R. Wasielewski, M. P. Niemczyk, W. A. Svec and E. B. Pewitt, J. Am. Chem. Soc., 1985, 107, 1080-1082; (c) L. S. Fox, M. Kozik, J. R. Winkler and H. B. Gray, Science, 1990, 247, 1069-1071.

11 H. M. McConnell, J. Chem. Phys., 1961, 35, 508-515.

12 (a) P. P. Edwards, H. B. Gray, M. T. J. Lodge and R. J. P. Williams, Angew. Chem., Int. Ed., 2008, 47, 6758-6765; (b) H. B. Gray and J. R. Winkler, Annu. Rev. Biochem., 1996, 65, 537-561; (c) E. A. Weiss, M. J. Ahrens, L. E. Sinks, A. V. Gusev, M. A. Ratner and M. R. Wasielewski, J. Am. Chem. Soc., 2004, 126, 5577-5584; (d) O. S. Wenger, B. S. Leigh, R. M. Villahermosa, H. B. Gray and J. R. Winkler, Science, 2005, 307, 99-102; (e) M. T. Indelli, C. Chiorboli, L. Flamigni, L. De Cola and F. Scandola, Inorg. Chem., 2007, 46, 5630-5641; $(f)$ S. Welter, F. Lafolet, E. Cecchetto, F. Vergeer and L. De Cola, ChemPhysChem, 2005, 6, 2417-2427.

13 (a) M. P. Eng and B. Albinsson, Angew. Chem., Int. Ed., 2006, 45, 5626-5629; (b) O. S. Wenger, Acc. Chem. Res., 2011, 44, 25-35; (c) K. Pettersson, J. Wiberg, T. Ljungdahl, J. Mårtensson and B. Albinsson, J. Phys. Chem. A, 2006, 110, 319-326; (d) J. Chen and O. S. Wenger, Chem. Sci., 2015, 6, 3582-3592; (e) S. F. Nelsen, R. F. Ismagilov and D. R. Powell, J. Am. Chem. Soc., 1998, 120, 1924-1925.

14 C. Atienza-Castellanos, M. Wielopolski, D. M. Guldi, C. van der Pol, M. R. Bryce, S. Filippone and N. Martín, Chem. Commun., 2007, 5164-5166.

15 (a) S. S. Isied, A. Vassilian, J. F. Wishart, C. Creutz, H. A. Schwarz and N. Sutin, J. Am. Chem. Soc., 1988, 110, 635-637; (b) E. H. Yonemoto, R. L. Riley, Y. I. Kim, S. J. Atherton, R. H. Schmehl and T. E. Mallouk, J. Am. Chem. Soc., 1992, 114, 8081-8087; (c) E. H. Yonemoto, G. B. Saupe, R. H. Schmehl, S. M. Hubig, R. L. Riley, B. L. Iverson and T. E. Mallouk, J. Am. Chem. Soc., 1994, 116, 4786-4795.

16 J. G. Kirkwood and F. H. Westheimer, J. Chem. Phys., 1938, 6, 506-512.

17 M. Kuss-Petermann and O. S. Wenger, Chimia, 2016, 70, 177-181. 
18 (a) J. Jortner, J. Chem. Phys., 1976, 64, 4860-4867; (b) P. F. Barbara, T. J. Meyer and M. A. Ratner, J. Phys. Chem., 1996, 100, 13148-13168.

19 (a) M. R. Wasielewski, Chem. Rev., 1992, 92, 435-461; (b) V. Balzani, Electron transfer in chemistry, VCH Wiley, Weinheim, 2001.

20 M. Tachiya and S. Murata, J. Phys. Chem., 1992, 96, 8441-8444.

21 (a) J. Hankache and O. S. Wenger, Chem. Commun., 2011, 47, 10145-10147; (b) J. Hankache, M. Niemi, H. Lemmetyinen and O. S. Wenger, Inorg. Chem., 2012, 51, 6333-6344; (c) J. Hankache, M. Niemi, H. Lemmetyinen and O. S. Wenger, J. Phys. Chem. A, 2012, 116, 8159-8168; (d) J. Hankache and O. S. Wenger, Phys. Chem. Chem. Phys., 2012, 14, 2685-2692; (e) J. Hankache and O. S. Wenger, Chem. - Eur. J., 2012, 18, 6443-6447; $(f)$ M. Orazietti, M. Kuss-Petermann, P. Hamm and O. S. Wenger, Angew. Chem., Int. Ed., 2016, DOI: 10.1002/anie.201604030; ( $g$ ) A. G. Bonn, O. Yushchenko, E. Vauthey and O. S. Wenger, Inorg. Chem., 2016, 55, 2894.

22 M. Sjödin, S. Styring, B. Åkermark, L. C. Sun and L. Hammarström, J. Am. Chem. Soc., 2000, 122, 3932-3936.

23 (a) D. Hanss and O. S. Wenger, Eur. J. Inorg. Chem., 2009, 3778-3790; (b) D. Hanss and O. S. Wenger, Inorg. Chem.,
2008, 47, 9081-9084; (c) D. Hanss and O. S. Wenger, Inorg. Chem., 2009, 48, 671-680; (d) D. Hanss, M. E. Walther and O. S. Wenger, Coord. Chem. Rev., 2010, 254, 2584-2592; (e) J. Chen, M. Kuss-Petermann and O. S. Wenger, Chem. Eur. J., 2014, 20, 4098-4104; $(f)$ J. Chen, M. Kuss-Petermann and O. S. Wenger, J. Phys. Chem. B, 2015, 119, 2263-2273; $(g)$ M. KussPetermann, H. Wolf, D. Stalke and O. S. Wenger, J. Am. Chem. Soc., 2012, 134, 12844-12854; (h) M. E. Walther and O. S. Wenger, ChemPhysChem, 2009, 10, 1203-1206; (i) M. E. Walther, J. Grilj, D. Hanss, E. Vauthey and O. S. Wenger, Eur. J. Inorg. Chem., 2010, 4843-4850; ( $j$ ) D. Hanss, M. E. Walther and O. S. Wenger, Chem. Commun., 2010, 46, 7034-7036.

24 S. S. Isied, M. Y. Ogawa and J. F. Wishart, Chem. Rev., 1992, 92, 381-394.

25 S. S. Isied, A. Vassilian, R. H. Magnuson and H. A. Schwarz, J. Am. Chem. Soc., 1985, 107, 7432-7438.

26 A. Vassilian, J. F. Wishart, B. Vanhemelryck, H. Schwarz and S. S. Isied, J. Am. Chem. Soc., 1990, 112, 7278-7286.

27 M. Y. Ogawa, J. F. Wishart, Z. Y. Young, J. R. Miller and S. S. Isied, J. Phys. Chem., 1993, 97, 11456-11463.

28 T. Higashino, T. Yamada, M. Yamamoto, A. Furube, N. V. Tkachenko, T. Miura, Y. Kobori, R. Jono, K. Yamashita and H. Imahori, Angew. Chem., Int. Ed., 2016, 55, 629. 